\title{
A Developed Identities for the Enhancement Factor, the Spectral Variable and the Dissipation Parameter for Raman Bands From Semicontinuous Metal Films
}

\author{
A.M. Gadou \\ Faculty of Science, Mansoura University, Mansoura, Egypt
}

The behavior of enhanced Raman scattering for Raman active molecules on metallic surfaces have been investigated in view of a developed identities for the enhancement factors $G^{R s}$, the spectral variable $X$, and the dissipation parameter of a monomer $\delta$ for Raman bands. A good agreement of our results with published data indicating that our suggested identities are fruitful.

\section{Introduction}

Surface enhanced Raman scattering (SERS) is one of the most intriguing optical effects [1,2]. However the most effective SERS systems are collections of interacting particles [2]. A fractal cluster is a system of interacting material particles called monomers. Fractals are particles in colloidal solutions, rough surfaces, disordered layers on surfaces [3]. The fractal excitation coherence length (L) within which the excitation of monomers are strongly correlated is limited by $\mathrm{R}_{\mathrm{o}}<<\mathrm{L}<<\mathrm{R}_{\mathrm{c}}$, where $\mathrm{R}_{\mathrm{o}}<$, is a characteristic separation between nearest monomers, and $R_{c}$ is the total radius of the cluster [3,4].

The aim of the present article is to develop identities to obtain the parameters $\mathrm{G}^{\mathrm{Rs}}, \mathrm{X}$ and $\delta$ for Raman bands of Raman active molecules on metallic surfaces.

Theory: We now deal to derive an identity for the enhancement factor $\mathrm{G}^{\mathrm{Rs}}$ for a certain Raman signal of a semicontinuous metal film. Since the Raman active molecules are assumed to be uniformly distributed over the film. In this case $\mathrm{G}^{\mathrm{Rs}}$ is equal to the ratio of the integrated band intensity of the considered signal $I_{R}$ to that in the absence of metal grains $I_{R}^{\circ}[1,5]$.

$$
\therefore G^{R s}=I_{R} / I_{R}^{o}
$$


Since the major contribution to the enhancement is understood to originate from the large local fields that arise from optical excitation of surface plasmons [1,2]. The resulted local conductivity of the film takes either the metallic values $\sigma_{\mathrm{m}}$ on the metal grains or the dielectric values $\sigma_{\mathrm{d}}$ outside the metal grains inwhich $\sigma_{\mathrm{m}} \gg \sigma_{\mathrm{d}}$. In this case [5]: $\sigma_{\mathrm{m}}=\mathrm{i} \varepsilon_{\mathrm{m}} \omega / 4 \pi, \sigma_{\mathrm{d}}=\mathrm{i} \varepsilon_{\mathrm{d}} \omega$ $/ 4 \pi$, and $\omega$ : is the frequency of the incident wave.

A respective dependence of $\mathrm{I}_{\mathrm{R}}, \mathrm{I}_{\mathrm{R}}^{\circ}$ on $\sigma_{\mathbf{m}}$ and $\sigma_{\mathbf{d}}$ [5] has the consequence that $\boldsymbol{G}_{\boldsymbol{c} \boldsymbol{a} \boldsymbol{l}}^{\boldsymbol{R} \boldsymbol{S}}$ depends on the optical quantity $\frac{\varepsilon_{m_{1}}^{2}}{\varepsilon_{d} \varepsilon m_{2}} \quad \varepsilon_{\mathrm{m}}=\varepsilon_{\mathrm{m} 1}+\mathrm{i} \varepsilon_{\mathrm{m} 2}$ $\varepsilon_{\mathrm{m}}$ : The dielectric const, of the metal film.

Since $\mathrm{G}^{\mathrm{Rs}}$ depends on the local fields which in turn depend on the external field (or the excitation frequency $\mathrm{v}_{\mathrm{ex}}$ ) $[5,6]$. Inaddition the molecular transition frequency $\Delta v_{\mathrm{mn}}$ between two adjacent vibrational states (within the molecule in its electronic ground state) of respective quantum numbers $m, n[7,8,9]$ is defined as:

$$
\therefore \Delta v_{\mathrm{mn}}=\mathrm{v}_{\mathrm{ex}}-\mathrm{v}_{\mathrm{st}}
$$

$\mathrm{v}_{\mathrm{st}}$ : The frequency of the Stokes mode (which is a Raman mode with a scattered frequency $<\mathrm{v}_{\mathrm{ex}}$ ) Furthermore the surface palsmon modes -in which the enhancement process must be affected - are very sensitive to any variations on the surface boundary metal/ dielectric whereby a limited infinitesimal density of these modes occurs around a unit frequency term $I_{v}[10]$.

The above demonstrations have the consequence that $G^{\mathrm{Rs}}$ depends on the parameter $\mathrm{P}_{\mathrm{v}}$

$$
\mathrm{P} v=\frac{\Delta v m n}{I_{v}}
$$

Consequently $\mathrm{G}^{\mathrm{Rs}}$ could be expressed as: $\mathrm{G}^{\mathrm{RS}}=\frac{\varepsilon_{m 1}^{2} p_{v}}{\varepsilon_{d} \varepsilon_{m 2}}$

Before dealing with an identity for the parameter $\mathrm{X}$ we must first of all define it, through the relation [2,3]

$$
1 / x_{o}=-(X+i \delta)
$$

$x_{o}$ : Is the polarizability of a monomer on the investigated metal film. As shown below in Figs. $(1,2)$ a continuous increase of $\mathrm{G}^{\mathrm{Rs}}$ with increasing $\bar{\hbar}$ corresponding to continuous decrease of the dissipation factor $\delta$ as well as a 
decrease of the damping process of the surface plasma waves $[15,16]$. Hence the spectral variable $X$ could be then postulated in a way taken into account the mechanism of the propagation of the surface plasma waves.

In this case, the surface waves propagated along the boundary metal/dielectric are localized waves transport no energy away from the boundary $[15,17]$. In addition the surface fields decay exponentially normal to the surface inside and outside the plasma $[18,19]$. Notice that the parameters $X$ and $\delta$ are multiplied by $\mathrm{R}_{0}{ }^{3}$ to give dimensionless quantities [2] - From the above demonstrations $\boldsymbol{R}_{\mathbf{0}}^{3}|\mathbf{X}|$ could be represented as:

$$
\boldsymbol{R}_{\mathbf{0}}^{3}|\mathbf{X}|=\operatorname{Aexp} \frac{(h \omega-B)}{I}-\mathbf{I}=1 \mathrm{eV}
$$

A: Is a damping parameter

$\mathrm{A}=\sqrt{R_{0}^{3} \delta}$

B: Is an energetic parameter its behavior is shown in Fig. (4) as a function of $\bar{\lambda}$. A maximum value of $\mathrm{B}(3.195 \mathrm{eV})$ is observed near the region of interband transitions for silver $(\lambda \sim 350 \mathrm{~nm})$. A continuous decrease of B with increasing

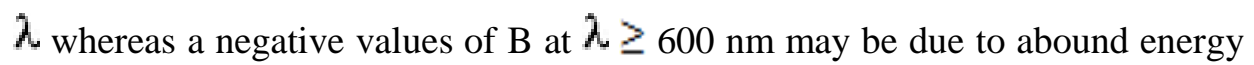
states near the region of the surface plasmon resonance condition which is accepted $[3,16,17]$.

In order to obtain experimental values of $\boldsymbol{R}_{\mathbf{0}}^{3} \mid \mathbf{X}$, a two identities of both $\boldsymbol{R}_{\mathbf{0}}^{\mathbf{3}}|\mathrm{X}|$, and $\boldsymbol{R}_{\mathbf{0}}^{\mathbf{3}} \delta$ have been derived from eq. 2 [2,3], whereas $\mathcal{X}_{\mathrm{o}}$ is given by [2]:

$$
x_{\mathrm{o}}=\boldsymbol{R}_{\boldsymbol{m}}^{3}\left(\varepsilon_{\mathrm{m}}-\varepsilon_{\mathrm{d}}\right) /\left(\varepsilon_{\mathrm{m}}+2 \varepsilon_{\mathrm{d}}\right)
$$

$\mathrm{R}_{\mathrm{m}}$ : Is the radius of the monomer.

Assuming $\mathrm{R}_{\mathrm{o}} \sim \mathrm{R}_{\mathrm{m}}$, one then obtains:

$$
\begin{aligned}
& \boldsymbol{R}_{\mathrm{o}}^{3}|\mathrm{X}| \approx\left[\frac{\varepsilon_{m 1}+3.54}{\varepsilon_{m 1}-1.77}\right] \\
& \boldsymbol{R}_{0}^{3} \delta=\frac{5.31 \varepsilon_{m 2}}{\left(\varepsilon_{m 1}-1.77\right) 2+\varepsilon^{2} m 2}
\end{aligned}
$$




\section{Consequently:}

$$
\left\langle\boldsymbol{R}_{\mathbf{0}}^{\mathbf{3}}|\mathrm{X}|\right\rangle \exp \approx\left|\frac{\left(\sqrt{R_{0}^{3} \delta}\right)_{\exp }\left(\varepsilon_{m 1}+3.54\right)}{\sqrt{5.31 \varepsilon_{m 2}}}\right|
$$

A calculated values of $\mathrm{G}^{\mathrm{Rs}}, \delta$ and $\mathrm{X}$ could be obtained by considering the Raman band $\Delta \mathrm{v}_{\mathrm{mn}}=1400 \mathrm{~cm}^{-1}$ for adsorbed citrate on colloidal silver - for the boundary $\mathrm{Ag} /$ water $\varepsilon_{\mathrm{d}}=1.77$ [13] whilst the values of $\varepsilon_{\mathrm{m} 1}, \varepsilon_{\mathrm{m} 2}$ were obtained from [14] - Hence using eq. 1 one obtains different values of $\mathrm{G}^{\mathrm{Rs}}$ for different excitation wavelengths.

Fig. (1) illustrates the relation between $\log \mathrm{G}^{\mathrm{RS}}$ and $\hbar$ using our calculated values of $\mathrm{G}^{\mathrm{RS}}$ and other values of published data $[13,20]$ The calculated as well as the experimental values of $\boldsymbol{R}_{\mathbf{0}}^{\mathbf{3}} \delta$ could be obtained from the corresponding values of $\mathrm{G}^{\mathrm{RS}}$ using the relation [2]: $\boldsymbol{R}_{\mathbf{0}}^{\mathbf{3}} \delta=1 /\left(\mathrm{G}^{\mathrm{Rs}}\right)^{1 / 3}$. Fig. (2).

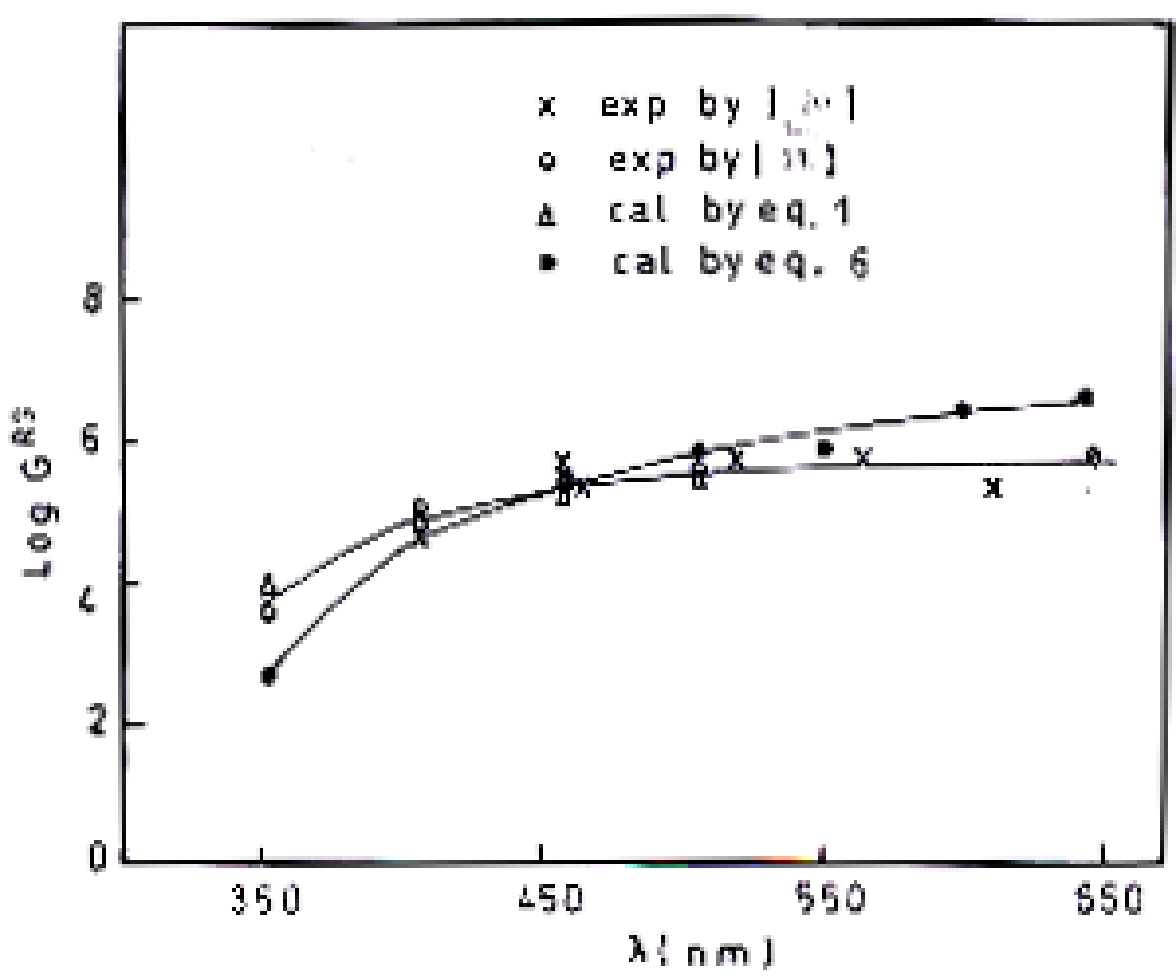

Fig.(1) : The variation of $\log \mathrm{G}^{\mathrm{Rs}}$ against the investigated wavelength $\lambda$ 


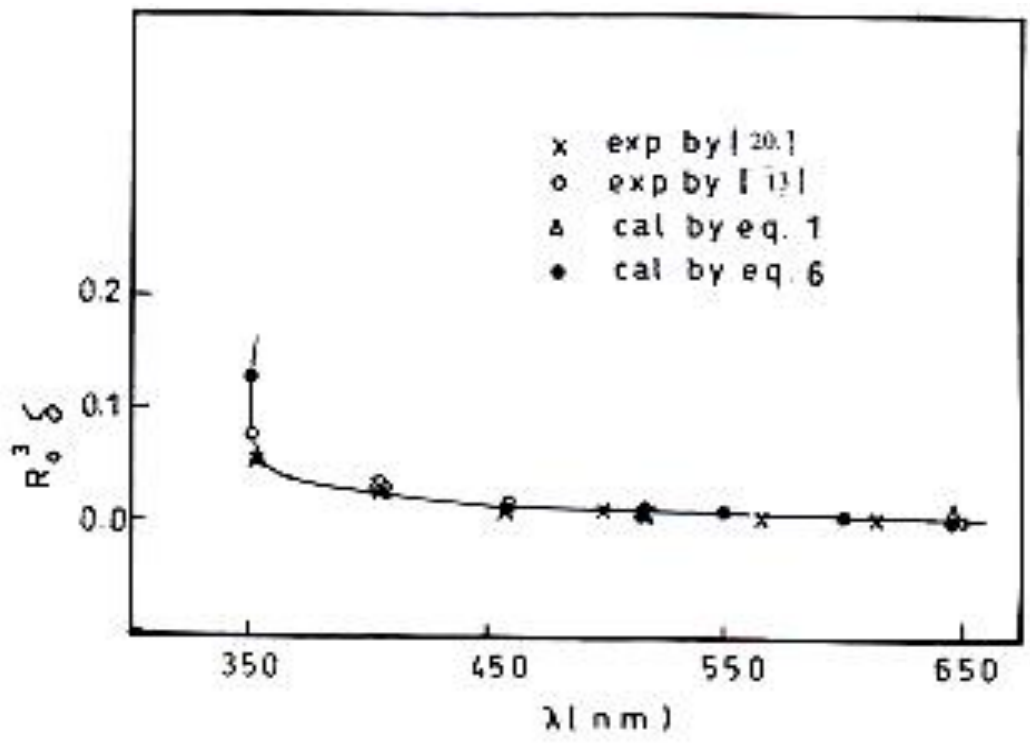

Fig.(2) : The variation of $\mathrm{R}_{0}{ }_{0} \delta$ against $\lambda$

shows the variation of $R_{0}^{3} \delta$ against $\lambda$ Fig. (3) reveals the spectral variable $R_{0}^{3}|\mathrm{x}|$ against $\lambda$ using the calculated values obtained from eqs $(3,5)$, the experimental values by $[13,20]$ with the help of eq. [7]. It is observed from Figs. (1-3) that the behavior for our calculated values of $\mathrm{G}^{\mathrm{Rs}}, R_{0}^{3} \delta$ and $R_{0}^{3}|\mathrm{x}|$ are in accordance with published data $[2,13,20]$, indicating that our suggested identities 1, 3 are useful and physically meaningful. It is observed from Fig. (3) that, the condition $R_{0}^{3}|\mathrm{x}| \sim 1$ corresponds to $\lambda \sim 6250 \mathrm{~A}^{\mathrm{o}}$ which nearly equals the resonance wavelength of the surface plasmons $\lambda,\left(6300 \mathrm{~A}^{0}\right)$ for the boundary $\mathrm{Ag} /$ water in accordance with the observations of [3].

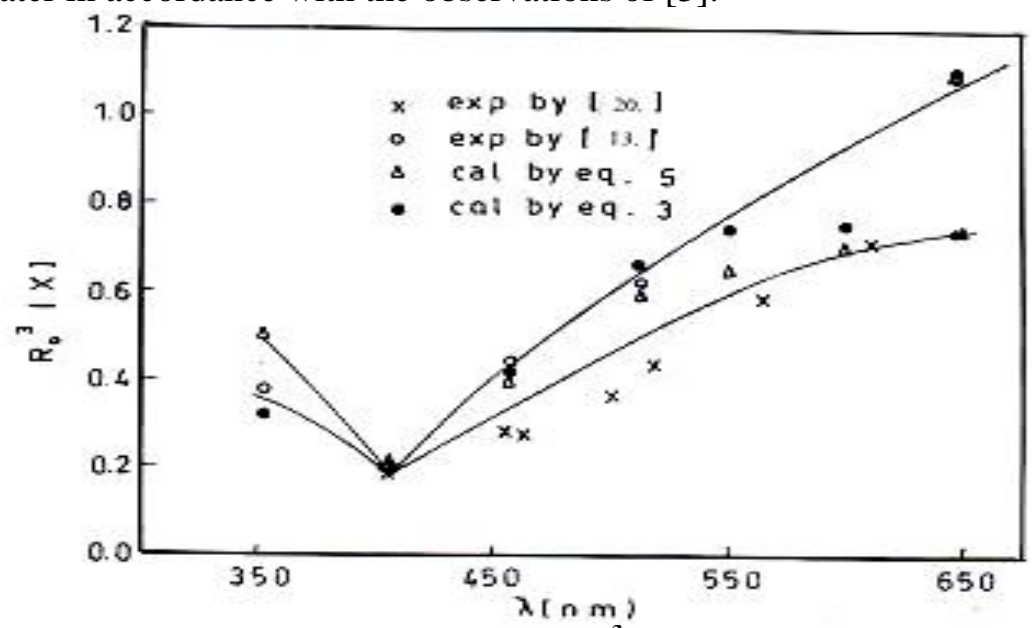

Fig.(3) : The variation of $R_{0}^{3}|\chi|$ against $\lambda$ 


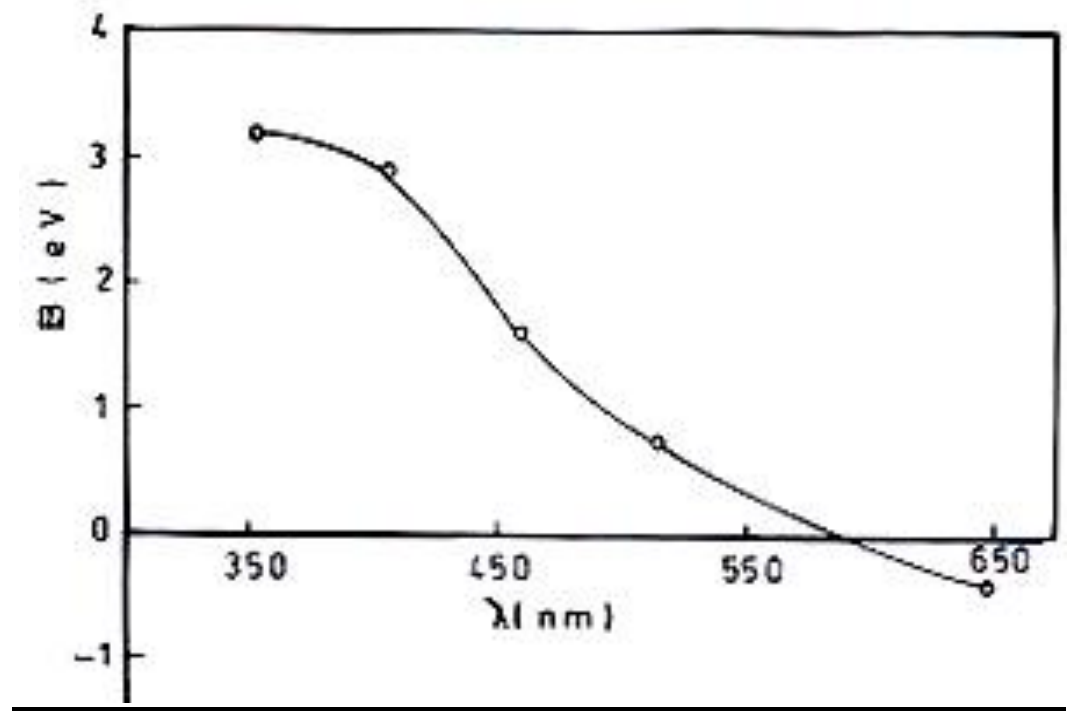

Fig.(4) : The variation of the energetic parameter $B$ against $\lambda$

\section{Conclusion:}

A developed identities for the three parameters $\mathrm{G}^{\mathrm{Rs}}, \mathrm{X}$ and $\delta$ have been obtained. At long wavelengths an increase of $G^{R s}$ is accompanied with a propagation of surface plasma waves as well as with a decrease of $\delta$. The condition $R_{0}^{3}|\mathrm{x}| \sim 1$ corresponds to surface plasmon resonance condition.

\section{Acknowledgement}

The author wold like to proceed his gratitude to Prof. Dr. H. Talaat, Ain Shams University, for fruitful discussions and critical reading of the manuscript.

\section{References}

1. M. Moskovits, Rev. Mod. Phys., 57, 783 (1985).

2. M.I. Stockman, Phys. Rev B Vol., 46, 2821 (1992).

3. V.A. Markel and L.S. Muratov, Phys. Rev., B. 43, 8183 (1991).

4. M.I. Stockman and F.G. Thomas, Phys. Rev., B 44,115 (1991).

5. F. Brouers and S. Blacher, Phys. Rev., B. 55, 13234 (1997).

6. A.K. Sarychev, V.M. Shalaev, Phys. Rep., 335, P 322 (2000). 
7. Vogel's, Textbook of Quantitative chemical analysis published by pearson Education (Singapore) Pte. Ltd, Indian Branch, 482 F.l.E. Paiparganj, Delhi 110092, India. P 696 - 698 (2004)

8. D.A. long. Raman Spectroscopy, McGraw - Hill International Book company, New York, P. 113 (1977).

9. Ira N. Levine Physical chemistry, International Edition ISBN 0-07-1122427. Exclusive rights by the McGraw-Hill companies, Inc, for manufacture and export P.774-776 (2002).

10. M.R. Philpott, J. chem. phys., 62, 1812 (1975).

11. R.J.H. Clark and R.E. Hester, Advances in infrared and Raman Spectroscopy, Vol. 5, Heyden \& Son Ltd, Spectrum House, Hillview Gardens, London, NW4 2JQ, pages 222 (1978).

12. A.C. Albrecht and M.C. Hutlly, J. Chem. Phys., 55, 4438 (1971).

13. M. Kerker, O. Siiman, L.A. Bumm and D.S. Wang. Appl. Opt., 19, 3253 (1980).

14. P.B. Johnson and R.W. Christy, Phys. Rev., B. 12, 4370 (1972).

15. A. Otto, Z. Physik, 216, p. 398 (1968).

16. E. Kretschmann, Z. Physik, 241, 313 (1971).

17. M.J. Jory, G.W. Bradberry, P.S. Cann and J.R. Sambles, Measurements Science \& Technology 6 [8], 1193, (1995).

18. H. Raether, Phys. Thin Films, 9, 145 (1977).

19. A. Otto Festkorper Probleme XIV, Experimental investigations of surface polaritons on plane interfaces p. 1-36 (1974).

20. O.Siiman, L.A. Bumm, B. Callaghan, C.G. Blatchford and M. Kerker, J. Phy. Chem., 87, 1014(1983). 